\title{
Existence of Faster Than Light Signals Implies Hypercomputation Already in Special Relativity ${ }^{\star}$
}

\author{
Péter Németi and Gergely Székely \\ Alfréd Rényi Institute of Mathematics \\ Mailing address: POB 127, H-1364 Budapest, Hungary \\ nemeti.peter@renyi.mta.hu, szekely.gergely@renyi.mta.hu
}

\begin{abstract}
Within an axiomatic framework, we investigate the possibility of hypercomputation in special relativity via faster than light signals. We formally show that hypercomputation is theoretically possible in special relativity if and only if there are faster than light signals.
\end{abstract}

Keywords: relativistic computation, special relativity, faster than light signals

\section{Introduction}

The theory of relativistic hypercomputation (i.e., the investigation of relativity theory based physical computational scenarios which are able to solve nonTuring-computable problems) has an extensive literature and it is investigated by several researchers in the past decades, see, e.g., 3], [5, [7, 9, 12, 15]. For an overview of different approaches to hypercomputation, see, e.g., [26].

It is well-known that hypercomputation is not possible in special relativity in the usual sense (i.e., the sense of Malament-Hogarth spacetimes), see, e.g., [12. In this paper, we show that it is possible to perform relativistic hypercomputation via ordinary computers (Turing machines) in special relativity if there are faster than light (FTL) signals, e.g., particles. We will also show that there have to be FTL signals if relativistic hypercomputation is possible in special relativity (via Turing machines), see Thm 2

It is interesting in and of itself to investigate the (logical) consequences of the assumption that FTL objects exist, independently of the question whether they really exist or not in our actual physical universe. Logic based axiomatic investigations typically aim for describing all the theoretically possible universes and not just our actual one. Moreover, so far we have not excluded the possibility of the existence of FTL entities in our actual universe; and from time to time there appear theories and experimental results suggesting the existence of FTL objects. Recently, the OPERA experiment, see [19], raised the interest in the possibility of FTL particles.

* This research is supported by the Hungarian Scientific Research Fund for basic research grants No. T81188 and No. PD84093. 
Contrary to the common belief, the existence of FTL particles does not lead to a logical contradiction within special relativity. For a formal axiomatic proof of this fact, see [28]. However, it is interesting to note that, in contrast with this result, the impossibility of the existence of FTL inertial observers follows from special relativity, see, e.g., [1].

The investigation of FTL motion in relativity theory goes back (at least) to Tolman, see, e.g., [31, p.54-55]. Since then a great many works dealing with FTL motion have appeared in the literature, see, e.g., [16], [18, [21, 22, [23], 25], 32 to mention only a few.

\section{Hypercomputation in SR}

It is well-known that we can send information back to the past if there are FTL particles, see, e.g., [28, 31, p.54-55]. It is natural to try using this possibility to design computers with greater computational power. We will show that uniformly accelerated relativistic computers can compute beyond the ChurchTuring barrier via using FTL signals. In this section, we show this fact informally. In Sect 5. we reconstruct our informal ideas of this section within an axiomatic theory of special relativity extended with accelerated observers.

Our first observation is that if we can send out an FTL signal with a certain speed, we also have to be able to send out arbitrarily fast signals, by the principle of relativity. Prop 1 is a formal statement of this observation. To informally justify this statement, let us assume that we can send out an FTL signal by a certain experiment, say with speed 1.01c. According to special relativity, for any FTL speed, say $10^{10} \mathrm{c}$, there is a inertial reference frame (moving relative to our frame) according to which our signal moves with this speed. By the principle of relativity, inertial frames are experimentally indistinguishable, see [10, $\S 5$, pp.149-159], [11, 30, pp.176-178]. So the experiment which is configured in our reference frame as our original experiment is seen by this moving inertial frame as yielding an FTL signal moving with speed $10^{10} \mathrm{c}$ in our frame. Therefore, in our (or any other inertial) reference frame, it is possible to send out an FTL signal with any speed.

Let us see the construction of our special relativistic hypercomputer. Let the computer be accelerated uniformly with respect to an inertial observer, see Fig 11 There is an event $O$ with the following property: any event $E$ on the worldline of our uniformly accelerated computer is simultaneous with $O$, according to the inertial observer comoving with the computer at $E$, see, e.g., [17, Fig.6.4, p.173], 20, Fig.5.13, p.152].

Now let us show that this configuration can be used to decide non-Turingcomputable questions if there are FTL signals. Let us set the computer to work on some recursively enumerable but non Turing-computable problem, say the decision problem for the consistency of ZF set theory; the computer enumerates one by one all the consequences of ZF. Let us fix an event $M$ on the worldline of

\footnotetext{
${ }^{1}$ In relativity theory, uniform acceleration means motion along a hyperbola (according to inertial observers), see, e.g., 6] §3.8, pp.37-38], [17 §6], 24, §12.4, pp.267-272].
} 


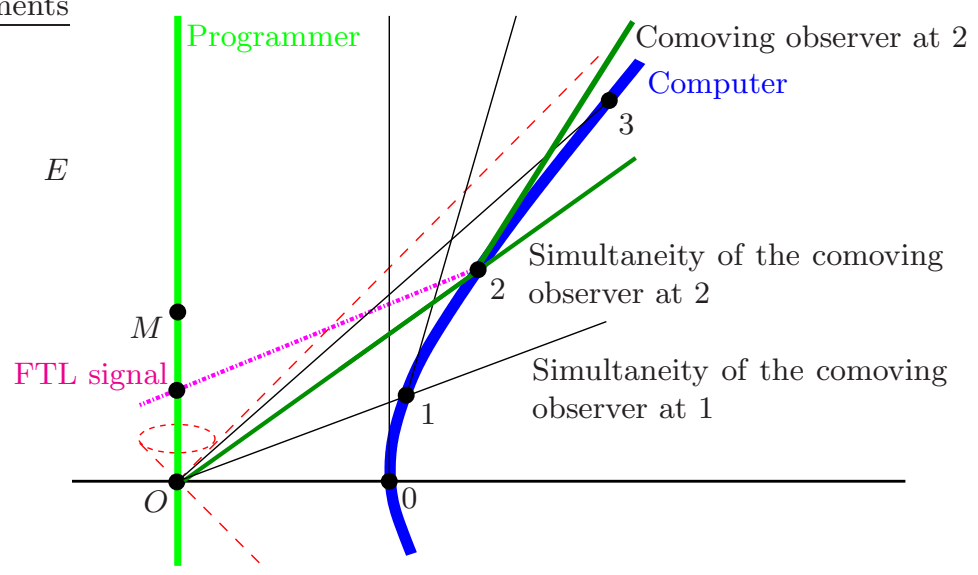

Fig. 1. Illustration of hypercomputation via FTL particles

the programmer which is later than $O$ according to him. Now, if the computer finds a contradiction, let it send out a fast enough signal which reaches the programmer before event $M$. Such signal exists since, by our first observation, the computer can send out a signal which is arbitrarily fast with respect to his coordinate system (i.e., any half line in the "upper" half space determined by the comoving observer's simultaneity can be the worldline of the signal). Therefore, if the programmer receives a signal between events $O$ and $M$, he knows that ZF is inconsistent; and if there is no signal between $M$ and $O$, he knows that the computer has not found any contradiction, so after event $M$ the programmer can conclude that there is no contradiction in ZF set theory. The same way, by this thought experiment using FTL signals, we can decide (experimentally) any recursively enumerable set of numbers.

If there are no FTL signals, then the whole computation has to happen in the causal past of the event when the programmer learns the result of computation. However, in special relativity, the computer remaining within the causal past of any event has only finite time to compute by the twin paradox theorem. That is why hypercomputation is not possible in special relativity without FTL signals. This argument is also the basis of proving that Minkowski spacetime is not a Malament-Hogarth spacetime.

\section{The Language of Our Axiom Systems}

To formalize the result of Sect 2 , we need an axiomatic theory of special relativity extended with accelerated observers. To introduce any axiomatic theory, first we have to fix the set of basic symbols of the theory, i.e., what objects and relations between them we will use as basic concepts. 
Here we will use the following two-sorted 2 language of first-order logic parametrized by a natural number $d \geq 2$ representing the dimension of spacetime:

$$
\{B, Q ; \mathrm{Ob}, \mathrm{IOb}, \mathrm{Ph},+, \cdot, \leq, \mathrm{W}\},
$$

where $B$ (bodies) and $Q$ (quantities) are the two sorts, Ob (observers), IOb (inertial observers) and $\mathrm{Ph}$ (light signals) are one-place relation symbols of sort $B,+$ and $\cdot$ are two-place function symbols of sort $Q, \leq$ is a two-place relation symbol of sort $Q$, and $\mathrm{W}$ (the worldview relation) is a $d+2$-place relation symbol the first two arguments of which are of sort $B$ and the rest are of sort $Q$.

Relations $\mathrm{Ob}(o), \operatorname{IOb}(m)$ and $\mathrm{Ph}(p)$ are translated as "o is an observer," " $m$ is an inertial observer," and " $p$ is a light signal," respectively. To speak about coordinatization, we translate $\mathrm{W}\left(k, b, x_{1}, x_{2}, \ldots, x_{d}\right)$ as "body $k$ coordinatizes body $b$ at space-time location $\left\langle x_{1}, x_{2}, \ldots, x_{d}\right\rangle$," (i.e., at space location $\left\langle x_{2}, \ldots, x_{d}\right\rangle$ and instant $\left.x_{1}\right)$.

Quantity terms are the variables of sort $Q$ and what can be built from them by using operations + and $\cdot$, body terms are only the variables of sort $B$. Relations $\mathrm{Ob}(o), \operatorname{lOb}(m), \mathrm{Ph}(p), \mathrm{W}\left(m, b, x_{1}, \ldots, x_{d}\right), x=y$, and $x \leq y$ where $o$, $m, p, b, x, y, x_{1}, \ldots, x_{d}$ are arbitrary terms of the respective sorts are so-called atomic formulas of our first-order logic language. Formulas are built up from these atomic formulas by using the logical connectives not $(\neg)$, and $(\wedge)$, or $(\vee)$, implies $(\rightarrow)$, if-and-only-if $(\leftrightarrow)$ and the quantifiers exists $(\exists)$ and for all $(\forall)$.

To make them easier to read, we omit the outermost universal quantifiers from the formalizations of our axioms, i.e., all the free variables are universally quantified.

We use the notation $Q^{d}$ for the set of all $d$-tuples of elements of $Q$. If $\bar{x} \in Q^{d}$, we assume that $\bar{x}=\left\langle x_{1}, \ldots, x_{d}\right\rangle$, i.e., $x_{i}$ denotes the $i$-th component of the $d$ tuple $\bar{x}$. Specially, we write $\mathrm{W}(m, b, \bar{x})$ in place of $\mathrm{W}\left(m, b, x_{1}, \ldots, x_{d}\right)$, and we write $\forall \bar{x}$ in place of $\forall x_{1} \ldots \forall x_{d}$, etc.

We use first-order set theory as a meta theory to speak about model theoretical terms, such as models. The models of this language are of the form

$$
\mathfrak{M}=\left\langle B, Q ; \mathrm{Ob}_{\mathfrak{M}}, \mathrm{IOb}_{\mathfrak{M}}, \mathrm{Ph}_{\mathfrak{M}},+_{\mathfrak{M}}, \cdot \mathfrak{M}, \leq_{\mathfrak{M}}, \mathrm{W}_{\mathfrak{M}}\right\rangle
$$

where $B$ and $Q$ are nonempty sets, $\mathrm{Ob}_{\mathfrak{M}}, \mathrm{IOb}_{\mathfrak{M}}$ and $\mathrm{Ph}_{\mathfrak{M}}$ are unary relations on $B,+_{\mathfrak{M}}$ and $\cdot \mathfrak{M}$ are binary functions and $\leq_{\mathfrak{M}}$ is a binary relation on $Q$, and $\mathrm{W}_{\mathfrak{M}}$ is a relation on $B \times B \times Q^{d}$. Formulas are interpreted in $\mathfrak{M}$ in the usual way. For the precise definition of the syntax and semantics of first-order logic, see, e.g., [4, §1.3], 86, §2.1, §2.2].

\section{Axioms of Special Relativity}

Let us recall some of our axioms for special relativity. Our first axiom states some basic properties of addition, multiplication and ordering true for real numbers.

\footnotetext{
${ }^{2}$ That our theory is two-sorted means only that there are two types of basic objects (bodies and quantities) as opposed to, e.g., Zermelo-Fraenkel set theory where there is only one type of basic objects (sets).
} 
AxOField: The quantity part $\langle Q,+, \cdot, \leq\rangle$ is an ordered field, i.e.,

$-\langle Q,+, \cdot\rangle$ is a field in the sense of abstract algebra; and

- the relation $\leq$ is a linear ordering on $Q$ such that

i) $x \leq y \rightarrow x+z \leq y+z$ and

ii) $0 \leq x \wedge 0 \leq y \rightarrow 0 \leq x y$ holds.

In the next axiom, we will use the concepts of time difference and spatial distance. The time difference of coordinate points $\bar{x}, \bar{y} \in Q^{d}$ is defined as:

$$
\operatorname{time}(\bar{x}, \bar{y}):=x_{1}-y_{1} \text {. }
$$

To speak about the spatial distance of any two coordinate points, we have to use squared distance since it is possible that the distance of two points is not amongst the quantities, e.g., the distance of points $\langle 0,0\rangle$ and $\langle 1,1\rangle$ is $\sqrt{2}$. So in the field of rational numbers, $\langle 0,0\rangle$ and $\langle 1,1\rangle$ do not have distance just squared distance. Therefore, we define the squared spatial distance of $\bar{x}, \bar{y} \in Q^{d}$ as:

$$
\operatorname{space}^{2}(\bar{x}, \bar{y}):=\left(x_{2}-y_{2}\right)^{2}+\ldots+\left(x_{d}-y_{d}\right)^{2} .
$$

Our next axiom is the key axiom of our axiom system of special relativity. This axiom is the outcome of the Michelson-Morley experiment, and it has been continuously tested ever since then. Nowadays it is tested by GPS technology.

AxPh: For any inertial observer, the speed of light is the same everywhere and

in every direction (and it is finite). Furthermore, it is possible to send out a light signal in any direction everywhere:

$$
\begin{aligned}
\operatorname{IOb}(m) \rightarrow \exists c_{m}\left(c_{m}>0 \wedge \forall \bar{x} \bar{y}[\right. & \operatorname{space}^{2}(\bar{x}, \bar{y})=c_{m}^{2} \cdot \operatorname{time}(\bar{x}, \bar{y})^{2} \\
\leftrightarrow & \exists[\operatorname{Ph}(p) \wedge \mathrm{W}(m, p, \bar{x}) \wedge \mathrm{W}(m, p, \bar{y})]]) .
\end{aligned}
$$

Let us note here that $\mathrm{AxPh}$ does not require (by itself) that the speed of light is the same for every inertial observer. It requires only that the speed of light according to a fixed inertial observer is a positive quantity which does not depend on the direction or the location. However, by $\mathrm{AxPh}$, we can define the speed of light according to inertial observer $m$ as the following binary relation:

$$
\begin{aligned}
\mathrm{c}(m, v) \stackrel{\text { def }}{\Longleftrightarrow} v>0 \wedge \forall \bar{x} \bar{y}[\exists p[\mathrm{Ph}(p) \wedge \mathrm{W}(m, p, \bar{x}) \wedge \mathrm{W}(m, p, \bar{y})] \\
\left.\rightarrow \operatorname{space}^{2}(\bar{x}, \bar{y})=v^{2} \cdot \operatorname{time}(\bar{x}, \bar{y})^{2}\right] .
\end{aligned}
$$

By $\mathrm{AxPh}$, there is one and only one speed $v$ for every inertial observer $m$ such that $\mathrm{c}(m, v)$ holds. From now on, we will denote this unique speed by $\mathrm{c}_{m}$.

Our next axiom connects the worldviews of different inertial observers by saying that they coordinatize the same "external" reality (the same set of events). By the event occurring for observer $m$ at coordinate point $\bar{x}$, we mean the set of bodies $m$ coordinatizes at $\bar{x}$ :

$$
\mathrm{ev}_{m}(\bar{x}):=\{b: \mathrm{W}(m, b, \bar{x})\}
$$


AxEv: All inertial observers coordinatize the same set of events:

$$
\operatorname{IOb}(m) \wedge \operatorname{IOb}(k) \rightarrow \exists \bar{y} \forall b[\mathrm{~W}(m, b, \bar{x}) \leftrightarrow \mathrm{W}(k, b, \bar{y})] .
$$

From now on, we will abbreviate the subformula $\forall b[\mathrm{~W}(m, b, \bar{x}) \leftrightarrow \mathrm{W}(k, b, \bar{y})]$ of AxEv to $\mathrm{ev}_{m}(\bar{x})=\mathrm{ev}_{k}(\bar{y})$. The next two axioms are only simplifying ones.

AxSelf: Any inertial observer is stationary relative to himself:

$$
\mathrm{IOb}(m) \rightarrow \forall \bar{x}\left[\mathrm{~W}(m, m, \bar{x}) \leftrightarrow x_{2}=\ldots=x_{d}=0\right] .
$$

Our last axiom on inertial observers is a symmetry axiom saying that they use the same units of measurement.

AxSymD: Any two inertial observers agree as to the spatial distance between two

events if these two events are simultaneous for both of them. Furthermore, the speed of light is 1 for all observers:

$$
\begin{aligned}
& \operatorname{IOb}(m) \wedge \operatorname{IOb}(k) \wedge x_{1}=y_{1} \wedge x_{1}^{\prime}=y_{1}^{\prime} \wedge \mathrm{ev}_{m}(\bar{x})=\operatorname{ev}_{k}\left(\bar{x}^{\prime}\right) \\
& \wedge \operatorname{ev}_{m}(\bar{y})=\operatorname{ev}_{k}\left(\bar{y}^{\prime}\right) \rightarrow \operatorname{space}^{2}(\bar{x}, \bar{y})=\operatorname{space}^{2}\left(\bar{x}^{\prime}, \bar{y}^{\prime}\right), \text { and } \\
& \operatorname{IOb}(m) \rightarrow \exists p[\operatorname{Ph}(p) \wedge \mathrm{W}(m, p, 0, \ldots, 0) \wedge \mathrm{W}(m, p, 1,1,0, \ldots, 0)] .
\end{aligned}
$$

Our axiom system SpecRel is the collection of the five simple axioms above:

$$
\text { SpecRel }:=\{\text { AxOField, AxPh, AxEv, AxSelf, AxSymD }\} .
$$

To show that SpecRel captures the kinematics of special relativity, let us introduce the worldview transformation between observers $m$ and $k$ (in symbols, $\mathrm{w}_{m k}$ ) as the binary relation on $Q^{d}$ connecting the coordinate points where $m$ and $k$ coordinatize the same (nonempty) events:

$$
\mathrm{w}_{m k}(\bar{x}, \bar{y}) \stackrel{d e f}{\Longleftrightarrow} \mathrm{ev}_{m}(\bar{x})=\mathrm{ev}_{k}(\bar{y}) \neq \emptyset .
$$

Map $P: Q^{d} \rightarrow Q^{d}$ is called a Poincaré transformation iff it is an affine bijection such that, for all $\bar{x}, \bar{y}, \bar{x}^{\prime}, \bar{y}^{\prime} \in Q^{d}$ for which $P(\bar{x})=\bar{x}^{\prime}$ and $P(\bar{y})=\bar{y}^{\prime}$,

$$
\operatorname{time}(\bar{x}, \bar{y})^{2}-\operatorname{space}^{2}(\bar{x}, \bar{y})=\operatorname{time}\left(\bar{x}^{\prime}, \bar{y}^{\prime}\right)^{2}-\operatorname{space}^{2}\left(\bar{x}^{\prime}, \bar{y}^{\prime}\right) .
$$

Thm 1 shows that our streamlined axiom system SpecRel perfectly captures the kinematics of special relativity since it implies that the worldview transformations between inertial observers are the same as in the standard non-axiomatic approaches. For the proof of Thm[1] see [2].

Theorem 1. Let $d \geq 3$. Assume SpecRel. Then $\mathrm{w}_{m k}$ is a Poincaré transformation if $m$ and $k$ are inertial observers.

The so-called worldline of body $b$ according to observer $m$ is defined as:

$$
\mathrm{wl}_{m}(b):=\{\bar{x}: \mathbf{W}(m, b, \bar{x})\}
$$


Corollary 1. Let $d \geq 3$. Assume SpecRel. The $\mathrm{wl}_{m}(k)$ is a straight line if $m$ and $k$ are inertial observers.

To extend SpecRel to accelerated observers, we need further axioms. We connect the worldviews of accelerated and inertial observers by the next axiom.

AxCmv: At each moment of its world-line, each observer coordinatizes the nearby world for a short while as an inertial observer does.

Axiom $\mathrm{AxCmv}$ is captured by formalizing the following statement: at each point of the worldline of an observer there is an inertial comoving observer such that the derivative of the worldview transformation between them is the identity map, see, e.g., [1] [27, §6] for details. We will also use the generalized (localized) versions of axioms AxEv and AxSelf of SpecRel assumed for every observer.

$\underline{\mathrm{AxEv}^{-}:}$Observers coordinatize all the events in which they participate:

$$
\mathrm{Ob}(k) \wedge \mathrm{W}(m, k, \bar{x}) \rightarrow \exists \bar{y} \mathrm{ev}_{m}(\bar{x})=\mathrm{ev}_{k}(\bar{y}) .
$$

AxSelf ${ }^{-}$: In his own worldview, the worldline of any observer is an interval of the time axis containing all the coordinate points of the time axis where the observer coordinatizes something:

$$
\begin{aligned}
& {\left[\mathrm{W}(m, m, \bar{x}) \rightarrow x_{2}=\ldots=x_{d}=0\right] \wedge} \\
& \quad\left[\mathrm{W}(m, m, \bar{y}) \wedge \mathrm{W}(m, m, \bar{z}) \wedge y_{1}<t<z_{1} \rightarrow \mathrm{W}(m, m, t, 0, \ldots, 0)\right] \wedge \\
& \exists b[\mathrm{~W}(m, b, t, 0, \ldots, 0) \rightarrow \mathrm{W}(m, m, t, 0, \ldots, 0)] .
\end{aligned}
$$

Let us add these three axioms to SpecRel to get a theory of accelerated observers:

$$
\text { AccRel }_{0}:=\text { SpecRel } \cup\left\{\mathrm{AxCmv}, \mathrm{AxEv}^{-}, \mathrm{AxSelf}^{-}\right\} .
$$

Since $\mathrm{AxCmv}$ ties the behavior of accelerated observers to the inertial ones and SpecRel captures the kinematics of special relativity perfectly by Thm 1, it is quite natural to think that $A c c R e I_{0}$ is a theory strong enough to prove the most fundamental theorems about accelerated observers. However, AccRel 0 does not even imply the most basic predictions of relativity theory about accelerated observers, such as the twin paradox. Moreover, it can be proved that even if we add the whole first-order logic theory of real numbers to AccRel ${ }_{0}$ is not enough to get a theory that implies (predicts) the twin paradox, see, e.g., [14, [27, §7].

In the models of $A c c R e l_{0}$ in which the twin paradox is not true, there are some definable gaps in W. Our next assumption excludes these gaps.

CONT: Every parametrically definable, bounded and nonempty subset of $Q$ has a supremum (i.e., least upper bound) with respect to $\leq$.

In CONT, "definable" means "definable in the language of AccRel, parametrically." CONT is Tarski's first-order logic version of Hilbert's continuity axiom in his axiomatization of geometry fitted to the language of AccRel. For a precise 
formulation of CONT, see [14, p.692] or [27, §10.1]. When $Q$ is the ordered field of real numbers, CONT is automatically true.

Let us extend AccRelo with axiom schema CONT:

$$
\text { AccRel }:=\text { AccRel }_{0} \cup \text { CONT. }
$$

It can be proved that AccRel implies the twin paradox, see [14, [27, §7.2].

That CONT requires the existence of supremum only for sets definable in AccRel instead of every set is important because it makes our postulate closer to the physical/empirical level. This is true because CONT does not speak about "any fancy subset" of the quantities, but just about those "physically meaningful" sets which can be defined in the language of our (physical) theory.

Let us now introduce some auxiliary axioms we will use here but not listed so far. To do so, let us call a linear bijection of $Q^{d}$ trivial transformation if leaves the time components (i.e., first coordinates) of coordinate points unchanged and it fixes the points of the time axis, i.e., the set of trivial transformation is:

Triv $:=\left\{T: T\right.$ is a linear bijection of $Q^{d}$,

$$
\left.T(\bar{y})_{1}=\bar{y}_{1} \text { and } T(\bar{x})=\bar{x} \text { if } \bar{x}_{s}=\bar{o}\right\},
$$

where $\bar{o}$ denotes the origin, i.e., coordinate point $\langle 0, \ldots, 0\rangle$.

AxThExp\# : Inertial observers can move with any speed less than the speed of light and new inertial reference frames can be constructed from other inertial reference frames by transforming them by trivial transformations and translations along the time axis 3

$$
\begin{array}{r}
\exists h \operatorname{IOb}(h) \wedge\left[\operatorname{IOb}(m) \wedge \operatorname{space}^{2}(\bar{x}, \bar{y})<\mathrm{c}_{m}^{2} \cdot \operatorname{time}(\bar{x}, \bar{y})^{2}\right. \\
\wedge T \in \operatorname{Triv} \rightarrow \exists k m^{\prime}\left[\operatorname{IOb}(k) \wedge \operatorname{IOb}\left(m^{\prime}\right) \wedge \mathrm{W}(m, k, \bar{x}) \wedge \mathrm{W}(m, k, \bar{y})\right. \\
\left.\left.\qquad \operatorname{ev}_{m}(\bar{x})=\operatorname{ev}_{k}(\bar{o}) \wedge \mathrm{w}_{m m^{\prime}}=T\right]\right] .
\end{array}
$$

The following axiom is a consequence of the principle of relativity. See [13, 28] for a formalization of the principle of relativity in our first-order logic language.

AxVel: If one observer can send out a body with a certain speed in a certain direction, then any other inertial observer can send out a body with this speed in this direction.

$$
\begin{aligned}
\operatorname{IOb}(m) \wedge & \operatorname{IOb}(k) \rightarrow \\
& {[\exists b[\mathrm{~W}(m, b, \bar{x}) \wedge \mathrm{W}(m, b, \bar{y})] \leftrightarrow \exists b[\mathrm{~W}(k, b, \bar{x}) \wedge \mathrm{W}(k, b, \bar{y})]] . }
\end{aligned}
$$

\footnotetext{
${ }^{3}$ Since linear bijections of $Q^{d}$ can be represented by a matrix of $d \times d$ quantities, the quantification $\forall T T \in$ Triv in AxTheExp\# can easily turned into a quantification over quantities.
} 
We call body $b$ inertial body iff there is an inertial observer $m$ according to who $b$ moves with uniform rectilinear motion:

$$
\begin{aligned}
\mathrm{IB}(b) \stackrel{\operatorname{def}}{\Longleftrightarrow} \exists m \bar{x} \bar{y}[\operatorname{IOb}(m) \wedge \bar{x} \neq \bar{y} \wedge \mathrm{W}(m, b, \bar{x}) \wedge \mathrm{W}(m, b, \bar{y}) \wedge \\
\forall \bar{z}(\mathrm{~W}(m, b, \bar{z}) \leftrightarrow \exists \lambda[\bar{z}=\bar{x}+\lambda(\bar{y}-\bar{x})])] .
\end{aligned}
$$

Let us now formulate the possibility of the existence of FTL inertial bodies. $\exists$ FTLBody: There is an inertial observer who can send out an FTL inertial body:

$$
\begin{array}{r}
\exists m b \bar{x} \bar{y}[\operatorname{IB}(b) \wedge \operatorname{IOb}(m) \wedge \mathrm{W}(m, b, \bar{x}) \wedge \mathrm{W}(m, b, \bar{y}) \wedge \\
\left.\quad \operatorname{space}^{2}(\bar{x}, \bar{y})>\mathrm{c}_{m}^{2} \cdot \operatorname{time}(\bar{x}, \bar{y})^{2}\right] .
\end{array}
$$

$\exists$ FTLBody implies that inertial observers can send out a body with arbitrary large speed in any direction if SpecRel, AxThExp\#, CONT and AxVel are assumed:

Proposition 1. Let $d \geq 3$. Assume SpecRel, AxThExp\#, CONT, AxVel and $\exists$ FTLBody. Then any inertial observer can send out a body with any speed in any direction:

$$
\mathrm{IOb}(m) \rightarrow \exists b[\mathrm{~W}(m, b, \bar{x}) \wedge \mathrm{W}(m, b, \bar{y})] .
$$

The proof of Prop 1 is in Sect 6 .

\section{Hypercomputation in AccRel}

In this section, we formulate our statement on the logical equivalence between the existence of FTL signals and the possibility of hypercomputation in special relativity as a theorem in our first-order logic language. To formulate the possibility of hypercomputation as a formula of our first-order logic language, let us define the life-curve $\mathrm{Ic}_{m}(k)$ of observer $k$ according to observer $m$ as the world-line of $k$ according to $m$ parametrized by the time measured by $k$, formally:

$$
\mathrm{Ic}_{m}(k):=\left\{\langle t, \bar{x}\rangle \in Q \times Q^{d}: \exists \bar{y} k \in \operatorname{ev}_{k}(\bar{y})=\operatorname{ev}_{m}(\bar{x}) \wedge y_{1}=t\right\} .
$$

The range and domain of a binary relation $R$, is defined as:

$$
\operatorname{Ran} R:=\{y: \exists x R(x, y)\} \text { and } \operatorname{Dom} R:=\{x: \exists y R(x, y)\} .
$$

The following formula of our language captures the possibility of relativistic hypercomputation in the sense used in the theory of relativistic computation.

HypComp: There are two observers a programmer $p$ and a computer $c$ and an instant $\tau$ in the programmer's worldline such that the computer has infinite time to compute, and during its computation the computer can send a signal $s_{t}$ to the programmer which reaches the programmer before the fixed instant:

$$
\begin{aligned}
& \exists p c \tau\left[\mathrm { Ob } ( p ) \wedge \mathrm { Ob } ( c ) \wedge \forall m x \left(\operatorname{IOb}(m) \wedge x \geq 0 \rightarrow x \in D o m \mid \mathbf{c}_{m}(c) \wedge\right.\right. \\
& \left.\left.\forall t\left[t>0 \rightarrow \exists t^{\prime} s_{t}\left[0<t^{\prime}<\tau \wedge s_{t} \in \mathrm{ev}_{m}\left(\operatorname{lc}_{m}(c)(t)\right) \cap \mathrm{ev}_{m}\left(\operatorname{lc}_{m}(p)\left(t^{\prime}\right)\right)\right]\right]\right)\right] .
\end{aligned}
$$


The following axiom ensures the existence of uniformly accelerated observers.

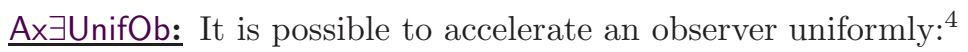

$$
\begin{aligned}
\mathrm{IOb}(m) \rightarrow & \exists k\left[\mathrm{Ob}(k) \wedge \operatorname{Dom} \mathbf{| c}_{m}(k)=Q\right. \\
& \left.\wedge \forall \bar{x}\left[\bar{x} \in \operatorname{Ran} \mathbf{c}_{m}(k) \leftrightarrow x_{2}^{2}-x_{1}^{2}=a^{2} \wedge x_{3}=\ldots=x_{d}=0\right]\right]
\end{aligned}
$$

Now we can state our theorem on the logical equivalence between the existence of FTL signals and the possibility of hypercomputation in special relativity:

Theorem 2. Let $d \geq 3$. Then

$$
\left\{\text { AccRel, AxThExp }{ }^{\#}, \text { Ax } \exists \text { Unifob, AxVel }\right\} \models \exists \text { FTLBody } \leftrightarrow \text { HypComp. }
$$

The proof of Thm 2 is in Sect 6 .

\section{Proofs}

In this section, we prove Prop 1 and Thm 2 .

Proof (Proof of Prop 1). Let $m$ be an inertial observer and let $\bar{x}, \bar{y} \in Q^{d}$. By AxSymDist, $\mathrm{c}_{m}=1$. If $\operatorname{space}^{2}(\bar{x}, \bar{y})<\operatorname{time}(\bar{x}, \bar{y})^{2}$, then there is a body (moreover, an inertial observer) $k$ such that $\mathrm{W}(m, k, \bar{x})$ and $\mathrm{W}(m, k, \bar{y})$ by AxTheExp\#. If $\operatorname{space}^{2}(\bar{x}, \bar{y})=\operatorname{time}(\bar{x}, \bar{y})^{2}$, then there is a body (moreover, a light signal) $p$ such that $\mathrm{W}(m, p, \bar{x})$ and $\mathbf{W}(m, p, \bar{y})$ by AxPh. So we only have to show that there is a body $b$ such that $\mathrm{W}(m, b, \bar{x})$ and $\mathbf{W}(m, b, \bar{y})$ if $\operatorname{space}^{2}(\bar{x}, \bar{y})>\operatorname{time}(\bar{x}, \bar{y})^{2}$. By $\exists$ FTLBody, there is an inertial observer who can send out an inertial body $b$ with a certain speed which is faster than the speed of light. By CONT, the quantity structure $\langle Q,+, \cdot, \leq\rangle$ is a real closed field, see [27, Prop.10.1.2]. Specially every positive number has a square root. Therefore, by AxThExp\#, we can rotate the worldview of any observer around the time axis by an arbitrary angle; and by Thm 1 and axiom AxThExp\#, there is an inertial observer whose simultaneity is so slanted that he sees $b$ moving with speed $\sqrt{\operatorname{space}^{2}(\bar{x}, \bar{y}) / \operatorname{time}(\bar{x}, \bar{y})^{2}}$. Consequently, there is an inertial observer who coordinatizes inertial body $b$ moving through $\bar{x}$ and $\bar{y}$. Then, by AxVel, every inertial observer can send out a body moving through $\bar{x}$ and $\bar{y}$.

Proof (Proof of Thm圆). Assume AccRel, AxThExp\#, AxヨUnifob, AxVel, and $\exists$ FTLBody. We have to prove HypComp. Let $p$ be an arbitrary inertial observer. Let $\tau=1$. Let $c$ be a uniformly accelerated observer such that $\mathrm{W}(p, c, \bar{x})$ iff $x_{2}^{2}-x_{1}^{2}=1$ and $x_{3}=\ldots=x_{d}=0$, see Fig 1 This observer $c$ exists and $\operatorname{Dom} \operatorname{lc}_{p}(c)=Q$ by $\mathrm{Ax} \exists$ UnifOb and Prop 3 below. By Prop 2 below, the simultaneity of any comoving observer $k$ of $c$ at the event of their meeting goes through

\footnotetext{
${ }^{4}$ In relativity theory, uniformly accelerated observers are moving along hyperbolas, see, e.g., 6] §3.8, pp.37-38], [17, §6], 24] §12.4, pp.267-272].
} 
the origin. So by Prop $k$ any comoving observer of $c$ (and thus $c$ ) can send out a body reaching $p$ before $M=\mathrm{Ic}_{p}(p)(\tau)$ and after $O=\mathrm{Ic}_{p}(p)(0)$, i.e.,

$$
\forall t\left[t>0 \rightarrow \exists t^{\prime} s_{t}\left[0<t^{\prime}<\tau \wedge s_{t} \in \mathrm{ev}_{p}\left(\operatorname{Ic}_{p}(c)(t)\right) \cap \operatorname{ev}_{p}\left(\operatorname{lc}_{p}(p)\left(t^{\prime}\right)\right)\right]\right]
$$

Let now $m$ be an arbitrary inertial observer and $x \geq 0$. By Prop 2 below, $D o m\left|c_{m}(c)=D o m\right| c_{p}(c)$. Therefore, $x \in D o m \mid c_{m}(c)=Q$. Also by Prop 3, $\mathrm{ev}_{m}\left(\operatorname{Ic}_{m}(c)(t)\right)=\mathrm{ev}_{p}\left(\operatorname{Ic}_{p}(c)(t)\right)$ for all $t \in Q$. Therefore,

$$
s_{t} \in \mathrm{ev}_{m}\left(\mathrm{Ic}_{m}(c)(t)\right) \cap \mathrm{ev}_{m}\left(\operatorname{Ic}_{m}(p)\left(t^{\prime}\right)\right) \leftrightarrow s_{t} \in \mathrm{ev}_{p}\left(\operatorname{Ic}_{p}(c)(t)\right) \cap \mathrm{ev}_{p}\left(\operatorname{lc}_{p}(p)\left(t^{\prime}\right)\right)
$$

Consequently,

$$
\forall t\left[t>0 \rightarrow \exists t^{\prime} s_{t}\left[0<t^{\prime}<\tau \wedge s_{t} \in \mathrm{ev}_{m}\left(\operatorname{lc}_{m}(c)(t)\right) \cap \mathrm{ev}_{m}\left(\operatorname{lc}_{m}(p)\left(t^{\prime}\right)\right)\right]\right] .
$$

This completes the proof of HypComp.

To prove the converse direction, assume that both $\neg \exists$ FTLBody and HypComp hold. Let $p$ and $c$ be arbitrary observers, and $\tau$ be an arbitrary time instant such that HypComp holds for $p, c$ and $\tau$, see Fig 2 Since the computer can send a signal to the programmer during its life from any instant $t>0$ and and there are no FTL particles, $\left.\mathrm{wl}_{m}(c) \subseteq I^{-}\left(\mathrm{Ic}_{m}(p)(\tau)\right)\right)^{5}$ according to any inertial observer $m$. By the twin paradox theorem, see, e.g., [14, [27, Thm.7.2.2], $c$ maximizes its time if it moves along a straight line. From this fact, it is easy to see that the longest path in $I^{-}\left(\mathrm{Ic}_{m}(p)(\tau)\right)$ starting at $\mid \mathrm{c}_{m}(c)(0)$ is the line segment connecting $\mathrm{Ic}_{m}(c)(0)$ and $\mathbf{I} \mathbf{c}_{m}(p)(\tau)$. Since even this path is finite, $c$ has only a finite time to compute. Therefore, subformula $\forall x \geq 0 \rightarrow x \in D o m \mid \mathrm{c}_{m}(c)$ of HypComp cannot be true. This contradiction proves our statement.

Proposition 2. Let $d \geq 3$. Assume AccRel. Let $p$ be an inertial observer and $c$ be a uniformly accelerated observer such that $\mathrm{W}(p, c, \bar{x})$ iff $x_{2}^{2}-x_{1}^{2}=a^{2}$ and $x_{3}=\ldots=x_{d}=0$ for some $0 \neq a \in Q$. Then the simultaneity of any comoving inertial observer $k$ of $c$ at $\bar{r}$ through $\bar{r}$, i.e., $\left\{\bar{y}: \mathrm{w}_{m k}(\bar{y})_{t}=\mathrm{w}_{m k}(\bar{r})_{t}\right\}$, contains the origin $\bar{o}$.

Proof. Let $p$ be an inertial observer, $c$ be a uniformly accelerated observer, $\bar{r}=$ $\left\langle r_{1}, r_{2}, 0, \ldots, 0\right\rangle$ be a point in the world-line of $c$, and $k$ be an inertial comoving observer of $c$ at $\bar{r}$. By Thm, $1, \mathrm{w}_{m k}$ is a Poincaré transformation. Therefore, the simultaneity of $k$ is Minkowski-orthogonal to his worldline, i.e.,

$$
\begin{aligned}
& \forall \bar{x} \bar{y} \bar{z}\left[\mathrm{~W}(m, k, \bar{x}) \wedge \mathrm{W}(m, k, \bar{z}) \wedge \bar{z} \neq \bar{x} \rightarrow\left[\mathrm{w}_{m k}(\bar{x})_{t}=\mathrm{w}_{m k}(\bar{y})_{t} \leftrightarrow\right.\right. \\
& \left.\left.\quad\left(y_{1}-x_{1}\right)\left(z_{1}-x_{1}\right)=\left(y_{2}-x_{2}\right)\left(z_{2}-x_{2}\right)+\ldots+\left(y_{d}-x_{d}\right)\left(z_{d}-x_{d}\right)\right]\right] .
\end{aligned}
$$

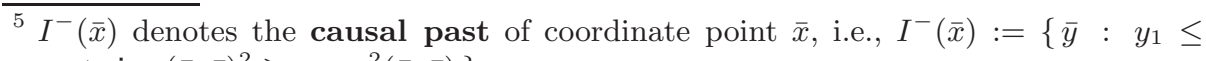
$\left.x_{1} \wedge \operatorname{time}(\bar{x}, \bar{y})^{2} \geq \operatorname{space}^{2}(\bar{x}, \bar{y})\right\}$. 


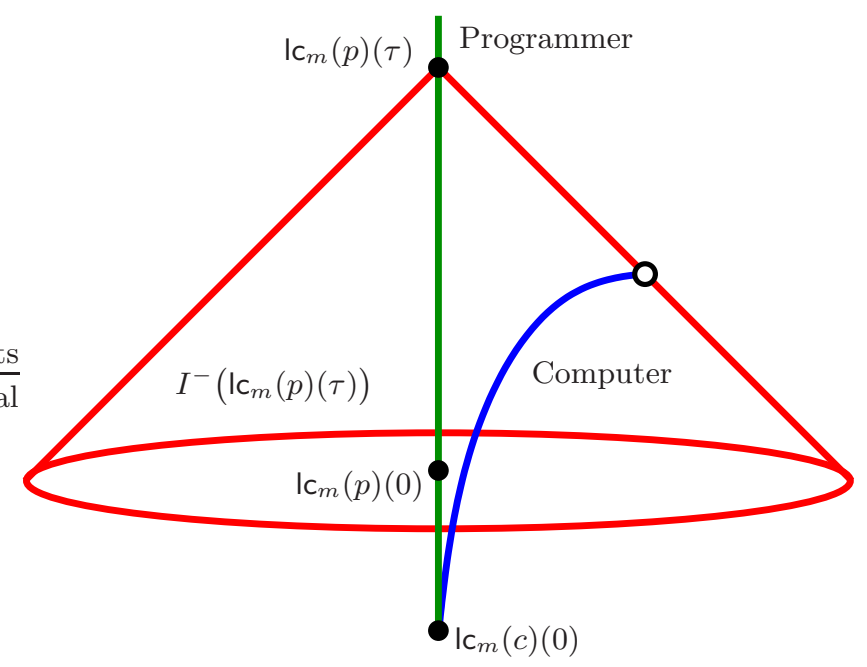

Fig. 2. Illustration for the proof of Thm 2

Therefore, we have to show that line $\bar{o} \bar{r}$ is Minkowski-orthogonal to $\mathrm{wl}_{m}(k)$. By AxCmv, the worldline of $k$ is the tangent line of the worldline of $c$ at $\bar{r}$. Therefore, by Lem 1 below, $\mathrm{wl}_{m}(k)=\left\{\bar{x}: r_{2} x_{2}=r_{1} x_{1}+a^{2}, x_{3}=\ldots=x_{d}=0\right\}$. Let $\bar{z}$ be a point of $\mathrm{wl}_{m}(k)$ different from $\bar{r}$. We have to show that $\left(0-r_{1}\right)\left(z_{1}-r_{1}\right)=$ $\left(0-r_{2}\right)\left(z_{2}-r_{2}\right)$. This equation is the same as $r_{2} z_{2}-r_{1} z_{1}=r_{2}^{2}-r_{1}^{2}$, which follows straightforwardly from $r_{2} z_{2}=r_{1} z_{1}+a^{2}$, i.e., $\bar{z} \in \mathrm{wl}_{m}(k)$, and $r_{2}^{2}=r_{1}^{2}+a^{2}$, i.e., $\bar{z} \in \mathrm{wl}_{m}(k)$. Thus line $\bar{o} \bar{r}$ is Minkowski-orthogonal to $\mathrm{wl}_{m}(k)$; and this is what we wanted to prove.

Lemma 1. Assume AxOField and CONT. The tangent line of hyperbola

$$
\left\{\bar{x}: x_{2}^{2}-x_{1}^{2}=a^{2}, x_{3}=\ldots=x_{d}=0\right\}
$$

at its point $\left\langle r_{1}, r_{2}, 0, \ldots, 0\right\rangle$ is

$$
\left\{\bar{x}: r_{2} x_{2}=r_{1} x_{1}+a^{2}, x_{3}=\ldots=x_{d}=0\right\} .
$$

Proof. Axioms AxOField and CONT imply that $Q$ is a real closed field, see 27, Prop.10.1.2]. By Tarski's theorem, real closed fields are elementarily equivalent, see [29]. Thus something which is expressible in the language of ordered fields is true in a real closed field iff it is true in the field of real numbers. The statement of this lemma can be formalized in the language of ordered fields and it is straightforward to show it in the ordered field of real numbers. Therefore, by Tarski's theorem, the statement is true in every model of AxOField and CONT; and this is what we waned to prove.

The following can be proved about life-curves, see [27, Prop. 6.1.6]. 
Proposition 3. Let $m, k$ and $h$ be observers. Then

1. $\mathrm{wl}_{m}(k)=\operatorname{Ran} \mathrm{Ic}_{m}(k)$ if $\mathrm{AxEv}^{-}$is assumed.

2. $\mathrm{Ic}_{m}(k)$ is a function if $\mathrm{AxPh}$ and $\mathrm{AxSelf}^{-}$are assumed and $m$ is an inertial observer.

3. Dom $\operatorname{lc}_{m}(h)=D o m \mid \mathbf{c}_{k}(h)$ and $\mathrm{ev}_{m}\left(\operatorname{Ic}_{m}(h)(t)\right)=\mathrm{ev}_{k}\left(\operatorname{Ic}_{k}(h)(t)\right)$ holds for all $t \in D o m \mid c_{m}(h)$ if $m$ and $k$ are inertial observers and AxEv is assumed.

\section{Concluding Remarks}

We have shown that, in special relativity, the possibility of hypercomputation is equivalent to the existence of FTL signals. A natural continuation is to investigate the question concerning the limits of the possibility of using FTL particles in hypercomputation in special and general relativity theories. For example, is there a natural assumption on spacetime which does not forbid the existence of FTL particles, but makes it impossible to use them for hypercomputation?

Of course our construction contains several engineering difficulties. For example, the larger the distance the more difficult to aim with a signal. Therefore, the computer has to calculate the speed of the FTL signal more and more accurately to ensure that the signal arrives to the programmer between events $O$ and $M$, see Fig 1 Thus the computer has to be able to aim with the FTL signal with arbitrary precision.

\section{References}

1. Andréka, H., Madarász, J.X., Németi, I., Székely, G.: A logic road from special relativity to general relativity. Synthese pp. Online-first: 1-17 (2011)

2. Andréka, H., Madarász, J.X., Németi, I., Székely, G.: What are the numbers in which spacetime? (2012), arXiv:1204.1350

3. Andréka, H., Németi, I., Németi, P.: General relativistic hypercomputing and foundation of mathematics. Nat. Comput. 8(3), 499-516 (2009)

4. Chang, C.C., Keisler, H.J.: Model theory. North-Holland Publishing Co., Amsterdam (1990)

5. Dávid, G., Németi, I.: Relativistic computers and the Turing barrier. Appl. Math. Comput. 178(1), 118-142 (2006)

6. d'Inverno, R.: Introducing Einstein's relativity. Oxford University Press, New York (1992)

7. Earman, J., Norton, J.D.: Forever is a day: supertasks in Pitowsky and MalamentHogarth spacetimes. Philos. Sci. 60(1), 22-42 (1993)

8. Enderton, H.B.: A mathematical introduction to logic. Academic Press, New York (1972)

9. Etesi, G., Németi, I.: Non-Turing computations via Malament-Hogarth spacetimes. Internat. J. Theoret. Phys. 41(2), 341-370 (2002)

10. Friedman, M.: Foundations of Space-Time Theories. Relativistic Physics and Philosophy of Science. Princeton University Press, Princeton (1983) 
11. Galilei, G.: Dialogues Concerning Two New Sciences. Macmillan, New York (1914, First published in 1638), translated from the Italian and Latin into English by Henry Crew and Alfonso de Salvio. http://ebooks.adelaide.edu.au/g/galileo/dialogues/complete.html

12. Hogarth, M.L.: Does general relativity allow an observer to view an eternity in a finite time? Found. Phys. Lett. 5(2), 173-181 (1992)

13. Madarász, J.X.: Logic and Relativity (in the light of definability theory). Ph.D. thesis, Eötvös Loránd Univ., Budapest (2002), http://www.math-inst.hu/pub/algebraic-logic/Contents.html

14. Madarász, J.X., Németi, I., Székely, G.: Twin paradox and the logical foundation of relativity theory. Found. Phys. 36(5), 681-714 (2006)

15. Manchak, J.B.: On the possibility of supertasks in general relativity. Found. Phys. 40(3), 276-288 (2010)

16. Matolcsi, T., Rodrigues, Jr., W.A.: The geometry of space-time with superluminal phenomena. Algebras Groups Geom. 14(1), 1-16 (1997)

17. Misner, C.W., Thorne, K.S., Wheeler, J.A.: Gravitation. W. H. Freeman and Co., San Francisco (1973)

18. Mittelstaedt, P.: What if there are superluminal signals? The European Physical Journal B - Condensed Matter and Complex Systems 13, 353-355 (2000)

19. OPERA collaboration: Measurement of the neutrino velocity with the OPERA detector in the CNGS beam (2011), arXiv:1109.4897

20. Petkov, V.: Relativity and the nature of spacetime. Frontiers Collection, SpringerVerlag, Berlin, second edn. (2009)

21. Recami, E.: Tachyon kinematics and causality: a systematic thorough analysis of the tachyon causal paradoxes. Found. Phys. 17(3), 239-296 (1987)

22. Recami, E.: Superluminal motions? A bird's-eye view of the experimental situation. Found. Phys. 31, 1119-1135 (2001)

23. Recami, E., Fontana, F., Garavaglia, R.: Special relativity and superluminal motions: a discussion of some recent experiments. Internat. J. Modern Phys. A 15(18), 2793-2812 (2000)

24. Rindler, W.: Relativity. Special, general, and cosmological. Oxford University Press, New York, second edn. (2006),

25. Selleri, F.: Superluminal signals and the resolution of the causal paradox. Found. Phys. 36, 443-463 (2006)

26. Stannett, M.: The case for hypercomputation. Appl. Math. Comput. 178(1), 8-24 (2006)

27. Székely, G.: First-Order Logic Investigation of Relativity Theory with an Emphasis on Accelerated Observers. Ph.D. thesis, Eötvös Loránd Univ., Budapest (2009)

28. Székely, G.: The existence of superluminal particles is consistent with the kinematics of Einstein's special theory of relativity (2012), arXiv:1202.5790

29. Tarski, A.: A decision method for elementary algebra and geometry. University of California Press, Berkeley and Los Angeles, Calif. (1951)

30. Taylor, E.F., Wheeler, J.A.: Spacetime Physics. W. H. Freeman and Company, New York (1997)

31. Tolman, R.C.: The Theory of the Relativity of Motion. University of California, Berkely (1917)

32. Weinstein, S.: Superluminal signaling and relativity. Synthese 148, 381-399 (2006) 